\title{
A personal view
}

\section{Smoking and coronary artery bypass surgery}

It is unfortunate that the recent controversy about coronary bypass surgery for smokers has been seen by the media and much of the public as an ethical issue about discrimination by the medical profession against those with unhealthy lifestyles. Doctors have been accused of assuming the role of God and deciding on moral grounds who should and who should not receive the benefits of surgery. This is a gross misunderstanding of the position taken by those who are hesitant about offering surgery to smokers: I have yet to meet a cardiologist who considers that patients should be penalised because of their habits. At the time of writing the debate rumbles on and it is depressing to find radio programmes such as Today and the responsible newspapers still discussing the issue in these ethical terms rather than addressing the question whether smokers should be treated differently from nonsmokers because their response to treatment may be different. If, as some believe, the risks of coronary surgery in smokers outweigh the benefits, whereas in non-smokers the reverse is true, no responsible person would advocate surgery for smokers. The problem is, however, not so simple and it is essential that health professionals discuss the available data to determine the best treatment for smokers. They must also debate the difficult question whether the access to surgery of those who impose an excessive burden on the inevitably limited surgical and intensive care resources (as smokers may do) should be restricted. Of course, such a question relates not only to smokers but also to the obese, the elderly, those requiring re-operation, and those with other disorders, such as obstructive airways disease.

Several organisations interested in this problem have emphasised that the decision to offer coronary surgery should be left to clinical judgment. I share this view but am concerned about what we mean by this term. Clinical judgment is based on knowledge, experience, and prejudice; and knowledge is the most important factor. Unfortunately in this, as in so many other areas of clinical medicine, our knowledge is seriously deficient. Nonetheless, there is enough information to permit some conclusions.

\section{Smoking and coronary heart disease}

It has been established beyond reasonable doubt that:

- Cigarette smoking is a causal factor in coronary heart disease

- Apparently healthy people who stop smoking reduce their risk of developing coronary disease

- Stopping smoking reduces the risk of further coronary events in those with manifest coronary disease

- Smoking can aggravate angina and silent myocardial ischaemia

- Stopping smoking may relieve symptoms to such an extent that coronary surgery, which otherwise would have been indicated for severe angina, is no longer necessary.
The case for stopping smoking is incontrovertible. Providing help for smokers to do so is as important as any other aspect of the management of ischaemic heart disease. But many smokers, some of whom are truly addicted, find it impossible to give up the habit. It is therefore important to consider the benefits and risks of anaesthesia and surgery in smokers with angina that is unresponsive to medical treatment.

\section{What are the perioperative risks in smokers?}

There is good evidence that postoperative respiratory complications and wound infections are commoner in those who smoke up to the time of operation than in those who give up for at least two months beforehand. A study by Warner et al ${ }^{1}$ of 192 patients from the Mayo Clinic is especially relevant. The incidence of postoperative chest complications was much higher in those who smoked up to the time of the operation than in those who gave up at least 8 weeks beforehand. Most of the chest complications were minor, consisting mainly of bronchospasm requiring dilators, increased sputum, and a greater need for physiotherapy; there was no increase in mortality. Time in hospital was marginally but significantly increased from 8.3 to 8.7 days.

Two recent papers reviewed factors that influence perioperative morbidity and mortality in patients undergoing coronary bypass surgery. Higgins et al from the Cleveland Clinic analysed 29 preoperative factors in 5051 patients. ${ }^{2}$ They found that current cigarette smoking was not a risk factor. A similar finding was reported from the Veterans Administration in 8065 patients of whom 3379 were current smokers. ${ }^{3}$ Both studies, however, found that chronic obstructive pulmonary disease was a major risk factor for morbidity and mortality.

We can conclude that smoking increases the demands on intensive care in the immediate postoperative period but it does not have a major effect on mortality. Many of those smokers who have a difficult postoperative course suffer from obstructive airways disease; these patients and non-smokers with this disorder need special preoperative preparation.

\section{What are the long-term effects of current smoking on the results of surgery?}

The best evidence about the relative benefits of surgery to smokers and non-smokers inevitably comes from randomised controlled studies. In the European Coronary Surgery Study, cigarette smoking at baseline was found not to affect adversely the benefits of surgery in reducing mortality. ${ }^{4}$ A recent review of the data (Varnauskas E. Personal communication 1993) showed that 10 years after surgery smokers and non-smokers had similar reductions in mortality (fig). An overview of all the coronary surgery bypass surgery trials confirms this finding. 

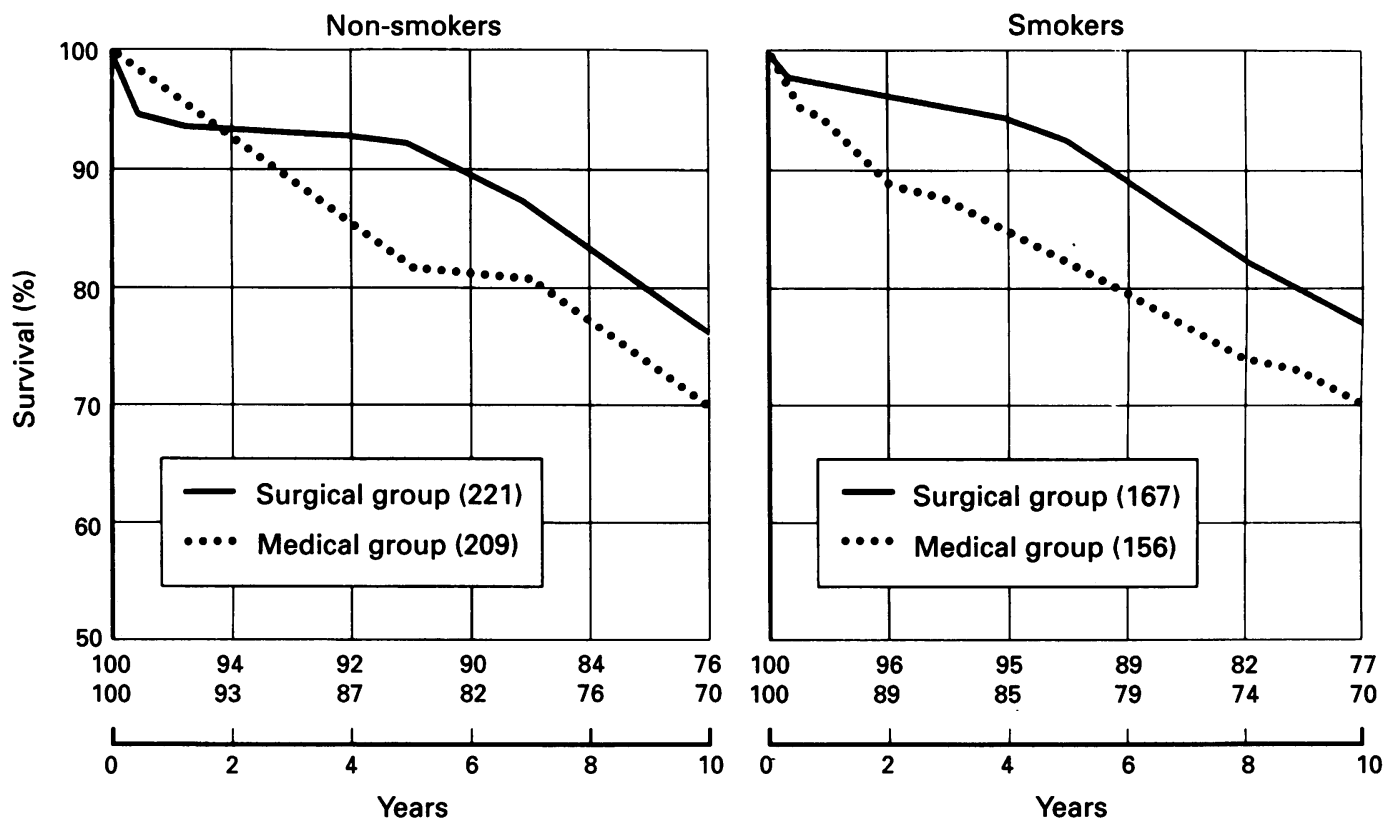

Comparative effects on survival after surgery and medical treatment in nonsmokers and smokers in the European Coronary

Surgery Study (Modified with permission from an article by $E$ Varnauskas published in

Läkartidningen

1993;90:4301).
These observations seem to conflict with the paper by Cavender $e t$ al in which the most striking finding was that survival at 10 years in 139 smokers randomised to surgery was $84 \%$ in those who quit smoking compared with $68 \%$ in those who did not. ${ }^{5}$ Those who continued to smoke suffered more angina, were more likely to be unemployed, and were admitted to hospital more often. It is postulated that the smoking increases the incidence of diseased and occluded grafts. ${ }^{67}$ Several aspects, however, make Cavender et al's study of limited value as a basis for decision-making:

- The observations concern the effect of giving up smoking after surgery in those who were smokers up to the time of surgery

- The observations are based on unrandomised subjects. Those who gave up smoking after surgery were compared with those who did not. The two groups are likely to have been different in other respects-smokers tend to have a poorer diet than non-smokers and to be less likely to comply with other aspects of treatment. This suggestion is supported by the fact that those who continued to smoke had higher cholesterol concentrations than those who quit $(P=0.006)$ and they were less likely to take $\beta$ blockers $(P=0.01)$. Information about aspirin usage was not provided

- A disturbing feature of the findings was that in the medically treated group non-quitters did as well as quitters. Cavender et al attempt to explain this by suggesting that the numbers in the study were too small to show a difference or that the more intensive medical treatmént in the medical group might have counteracted the adverse effects of smoking. If the study was too small, the validity of the observations in the equally small surgical group is also questionable. If medical treatment was not intensive enough, this is a clinical observation of fundamental importance and suggests that the results of surgery in the smokers would have been better if they had received better medical treatment.

A serious problem relates to the timing of surgery: if we delay surgery until the benefits of giving up smoking are achieved the patient may die in the intervening period. This raises two questions. Can we predict which patients will die in the near future unless they receive surgery and can cardiologists and cardiac surgeons do more to influence patients to give up smoking?

\section{Predicting early death in potential candidates for surgery}

It is generally agreed that smokers should be treated without delay if treatment is regarded as emergency or urgent. It is relatively easy to define the contexts in which emergency surgery is required. They include unstable angina unresponsive to medical treatment or failed angioplasty. It is more difficult to assess the risk of early death in other categories of patient. The severity of symptoms alone is a poor guide. Readily induced ischaemia, as demonstrated by exercise electrocardiography or nuclear imaging, provides important additional information both about prognosis and the potential benefits of surgery. In the presence of such findings, coronary angiography should be undertaken because it may show left main or proximal three vessel disease that warrants early operation on prognostic grounds. The urgency of surgery cannot be determined without such information. Of course, even with such information, prognosis remains difficult and as long as there are waiting lists there will always be unexpected deaths on the waiting list.

\section{Influence of the cardiologist, coronary} angiography, and surgery on smoking habit

Even though they know smoking is harmful many patients will not give up until advised to do so by someone they respect. Although I know of no scientific evidence to support this, personal experience and the anecdotal accounts of others convince me that cardiologists and cardiac surgeons can profoundly affect the behaviour of patients. Dramatic interventions and events certainly provide a powerful additional effect. Coronary angiography probably has some influence, and many patients give up after myocardial infarction and coronary surgery. In the CASS study about a third of smokers in both the medical and surgical groups gave up the habit in the succeeding years. ${ }^{8}$ In the European study there was a considerable reduction in smoking in the surgical group in the months after surgery; later the difference between the surgical and medical groups narrowed.

\section{Conclusion}

The data upon which decisions can be made about the 
role of surgery in smokers are inadequate and what data there are are open to interpretation. My prejudice/judgment is that smokers who have symptoms that are unresponsive to medical treatment should be assessed by a cardiologist, should undergo exercise testing and, depending on the results, should be considered for coronary angiography and possible surgery. Strenuous efforts should be made to help the smoker to give up the habit, but if lesions are found that are likely to be helped prognostically by surgery, this should be undertaken with the minimum of delay. If the coronary disease is not thought to have serious prognostic implications surgery should be delayed to give the patient more time to give up the habit, unless the symptoms are severely incapacitating, in which case surgery should be undertaken despite continued smoking. Programmes designed to help smokers with coronary disease quit the habit should be widely available. DESMOND G JULIAN
1 Warner MA, Offord KP, Lennon RL, Conover MA, Jansson-Schumacher $\mathrm{U}$. Role of preoperative cessation of smoking and other factors in postoperative pulmonary complications: a blinded prospective study of operative pulmonary complications: a blinded prospective study
coronary artery bypass patients. Mayo Clinic Proc 1989;64:609-16.

2 Higgins TL, Estafanous FG, Loop F, Beck G, Blum J, Paranandi L. Stratification of morbidity and mortality outcome by preoperative risk factors in coronary artery bypass patients. $\mathscr{f} A M A 1992 ; 267: 2344-8$.

3 Grover FL, Hammermeister KE, Burchfiel C. Initial report of the Veterans Administration preoperative risk assessment study for cardiac surgery. Ann Thorac Surg 1990;50:12-28.

4 European Coronary Surgery Study Group. Long-term results of prospective randomised study of coronary artery bypass surgery in stable angina pectoris. Lancet 1982;ii:1173-80.

5 Cavender JB, Rogers WJ, Fisher LD, Gersh BJ, Coggins CJ, Myers WO. Effects of smoking on survival and morbidity in patients randomized to medical or surgical therapy in the Coronary Artery Surgery Study (CASS): 10-year follow-up. $₹ \mathrm{Am}$ Coll Cardiol 1992;20:287-94.

6 Solymoss BC, Nadeau P, Millette D, Campeau L. Late thrombosis of saphenous vein coronary bypass grafts related to risk factors. Circulation 1988;78(suppl I):I-140-3.

7 FitzGibbon GM, Leach AJ, Kafka HP. Atherosclerosis of coronary artery bypass grafts and smoking. Can Med Assoc F 1987;136:45-7.

8 Rogers WJ, Coggin CJ, Gersh BJ, et al. Ten-year follow-up of quality of life in patients randomized to receive medical therapy or coronary bypass graft surgery: the Coronary Artery Surgery Study (CASS). bypass graft surgery: the Cor
Circulation 1990;82:1647-58. 